\title{
Mitral valve annuloplasty and papillary muscle relocation oriented by 3-dimensional transesophageal echocardiography for severe functional mitral regurgitation
}

\author{
Khalil Fattouch, MD, PhD, ${ }^{a}$ Giacomo Murana, MD, ${ }^{a}$ Sebastiano Castrovinci, MD, ${ }^{\text {a }}$ Claudia Mossuto, MD, ${ }^{\mathrm{b}}$ \\ Roberta Sampognaro, MD, ${ }^{\mathrm{c}}$ Maria Giuliana Borruso, MD, ${ }^{\mathrm{d}}$ Emanuela Clara Bertolino, MD, \\ Giuseppa Caccamo, MD, ${ }^{\mathrm{b}}$ Giovanni Ruvolo, $\mathrm{MD},{ }^{\mathrm{a}}$ and Patrizio Lancellotti, $\mathrm{MD}, \mathrm{PhD}^{\mathrm{e}}$
}

\begin{abstract}
Objective: The study of the mitral valve apparatus and its modifications during functional mitral regurgitation (FMR) is better revealed by 3-dimensional (3D) transesophageal echocardiography (TOE). To plan mitral valve repair by annuloplasty and papillary muscle (PPM) relocation, we proposed a valve repair procedure oriented by the new main features obtained by real-time 3D TOE reconstruction of the mitral valve apparatus.
\end{abstract}

\begin{abstract}
Methods: Since January 2008, 25 patients with severe FMR before mitral valve repair were examined. Mean coaptation depth and mean tenting area were $1.3 \pm 0.2 \mathrm{~cm}$ and $3.2 \pm 0.5 \mathrm{~cm}^{2}$, respectively. Intraoperative 2D and 3D TOE were performed, followed by a 3D offline reconstruction of the mitral valve apparatus. A schematic mitral valve apparatus model was obtained. A geometric model like a truncated cone was traced in according to the preoperative measurements. The size of the prosthetic ring was selected preoperatively according to the anterior leaflet surface. The expected truncated cone after annuloplasty was retraced. A conventional normal coaptation depth about $0.6 \mathrm{~cm}$ was used to detect the new position of the PPM tips.
\end{abstract}

Results: Offline reconstruction of the mitral valve apparatus and respective truncated cone were feasible in all patients. The expected position of the PPM tips desirable to reach a normal tenting area with a coaptation depth $0.6 \mathrm{~cm}$ or less was obtained in all patients. After surgery, all parameters were calculated and no statistically significant difference was found compared with the expected data.

Conclusions: PPM relocation plus ring annuloplasty reduce mitral valve tenting and may improve mitral valve repair results for patients with severe FMR. This technique may be easily and precisely guided by preoperative offline 3D echocardiographic mitral valve reconstruction. (J Thorac Cardiovasc Surg 2012;143:S38-42)

Functional mitral regurgitation (FMR) remains one of the most complex and unresolved aspects in the management of heart valve disease. Undersizing mitral valve annuloplasty with a prosthetic ring was first introduced by Bolling and associates ${ }^{1}$ and has recently become the preferred treatment for FMR. However, in several series this therapeutic approach has been associated with recurrence of mitral regurgitation (MR) in as much as $30 \%$ of patients at 6 months

From the Departments of Cardiac Surgery a and Internal Medicine and Cardiovascular Diseases, ${ }^{\mathrm{b}}$ University of Palermo, Palermo, Italy; The Department of Cardiology, ${ }^{\mathrm{c}}$ GVM Care and Research, Palermo, Italy; the Department of Cardiovascular Medicine, ${ }^{\mathrm{d}}$ San Raffaele Hospital, Milano, Italy; and the Heart Valve Clinic, ${ }^{\mathrm{e}}$ University of Liège, Department of Cardiology, CHU Sart Tilman, Liège, Belgium.

Disclosures: Khalil Fattouch, Giacomo Murana, Sebastiano Castrovinci, Claudia Mossuto, Roberta Sampognaro, Maria Giuliana Borruso, Emanuela Clara Bertolino, Giuseppa Caccamo, Giovanni Ruvolo, and Patrizio Lancellotti have nothing to disclose with regard to commercial support.

Presented at The American Association for Thoracic Surgery Mitral Conclave, New York, New York, May 5-6, 2011.

Received for publication May 14, 2011; revisions received Nov 22, 2011; accepted for publication Jan 4, 2012; available ahead of print Jan 30, 2012

Address for reprints: Khalil Fattouch, MD, PhD, University of Palermo, Department of Cardiac Surgery, Via Liborio Giuffré, 5, 90127, Palermo, Italy (E-mail: khalilfattouch@hotmail.com).

0022-5223/\$36.00

Copyright (c) 2012 by The American Association for Thoracic Surgery

doi:10.1016/j.jtcvs.2012.01.010 and $60 \%$ of patients 3 to 5 years after surgery. ${ }^{2,3}$ The underlying myocardiopathy in FMR may be ischemic or idiopathic. However, in both cases left ventricular (LV) remodeling leads to mitral valve annulus dilatation, flatness, papillary muscle (PPM) displacement, and mitral leaflet tethering. The pathologic 3-dimensional (3D) anatomy of FMR is complex and varies extensively between patients. The inconsistent clinical response to annuloplasty strongly suggests that there is significant variability in the main contributors to mitral valve incompetence in individual cases. The ability to determine the relative contribution of annular dilatation and leaflet tethering on FMR could help in making clinical decisions in favor of either isolated annuloplasty or more complex surgery combining repair and a subvalvular approach like PPM relocation. ${ }^{4}$ Displacement of the PPMs owing to LV remodeling is a key feature in the development of FMR. Therefore, a detailed preoperative 3D analysis of the entire mitral valve apparatus (MVA) is mandatory for planning surgical strategy. ${ }^{5}$ Real-time 3D echocardiography allows accurate evaluation of the MVA, including the LV chamber and PPM position. ${ }^{6}$ Recent development of matrix-array transducers has made real-time 3D echocardiography a clinically applicable tool and has affected mitral valve repair strategies. ${ }^{7}$ We postulated that in 

Abbreviations and Acronyms
$2 \mathrm{D}=2$-dimensional
FMR $=$ functional mitral regurgitation
$\mathrm{LV}=$ left ventricle (ventricular)
$\mathrm{MR}=$ mitral regurgitation
MVA $=$ mitral valve apparatus
PPM = papillary muscle
PTFE $=$ polytetrafluoroethylene
$\mathrm{TOE}=$ transesophageal echocardiography

selected patients with FMR a more comprehensive assessment of the 3D anatomy of the MVA using a dedicated mitral valve quantification software (QLab-MVQ; Philips Medical Systems, Andover, Mass) would represent an initial step toward a patient-specific repair approach. In the present study, we performed a mitral valve annuloplasty plus PPM relocation oriented by preoperative real-time 3D echocardiography.

\section{METHODS \\ Population}

Since January 2008, a total of 25 patients (mean age, $63 \pm 5$ years) with FMR were scheduled in our institution for mitral valve repair with or without coronary artery bypass grafting. There were 15 patients with ischemic and 10 with idiopathic cardiomyopathy. All patients were in a New York Heart Association functional class of more than II. Mean LV ejection fraction was $34 \% \pm 8 \%$. All patients had mitral annulus dilatation and symmetric leaflet tethering owing to PPM displacement (MR type I + IIIb of Carpentier's classification). Mean coaptation depth and mean tenting area were $1.3 \pm$
$0.2 \mathrm{~cm}$ and $3.2 \pm 0.5 \mathrm{~cm}^{2}$, respectively. Intraoperative 2-dimensional (2D) and real-time 3D transesophageal echocardiography (TOE) were performed, followed by an offline reconstruction of the MVA using QLab-MVQsoftware and QLab-3DQ advanced software (Philips Medical Systems).

\section{Real-Time 3D TOE Data Acquisition and Analysis}

TOE was performed using the iE33 ultrasound imaging system (Philips Medical Systems) equipped with the fully sampled matrix-array TOE transducer (X7-2t). The probe was positioned at the midesophageal level at a $0^{\circ}$ tilt (4-chamber view). Electrocardiographically gated full-volume images were acquired during 6 consecutive cardiac cycles. Care was taken to include the entire MVA throughout the acquisition. The images were acquired during a brief suspension of mechanical ventilation to avoid stitch artifacts, and special care was taken to stabilize the probe during data acquisition. All images were digitally stored for offline analysis with the MVQ software. This software helps to build a 3D model, step by step, of the MVA (annulus, anterior and posterior leaflets, coaptation line, and potential coaptation defects, as well as the angles between the mitral leaflets and the plane of the annulus). Moreover, the position of the PPMs was also evaluated.

\section{MVA Analysis With MVQ Software}

Off-line preoperative construction of the MVA. The measurements of the MVA included the intercommissural and anteroposterior annular diameters, the area of the anterior and posterior leaflets, the tenting area and volume, the coaptation depth, the angles between the mitral annulus and the mitral leaflets, and the distance between the interpapillary muscles. The interpapillary distance was measured between PPM tips; the distance between each papillary muscle tip and its ipsilateral and contralateral annulus was also calculated on multislice images view (Figure 1, A). The shape of the mitral valve annulus and the coaptation line between the leaflets were traced to have a schematic model of the mitral valve annulus and leaflets (Figure 1,B). A schematic model of the mitral valve apparatus was obtained by QLab-MVQ (Figure 2). A baseline truncated cone was constructed in all patients according to the preoperative anteroposterior and intercommissural annulus diameters, the distance between the

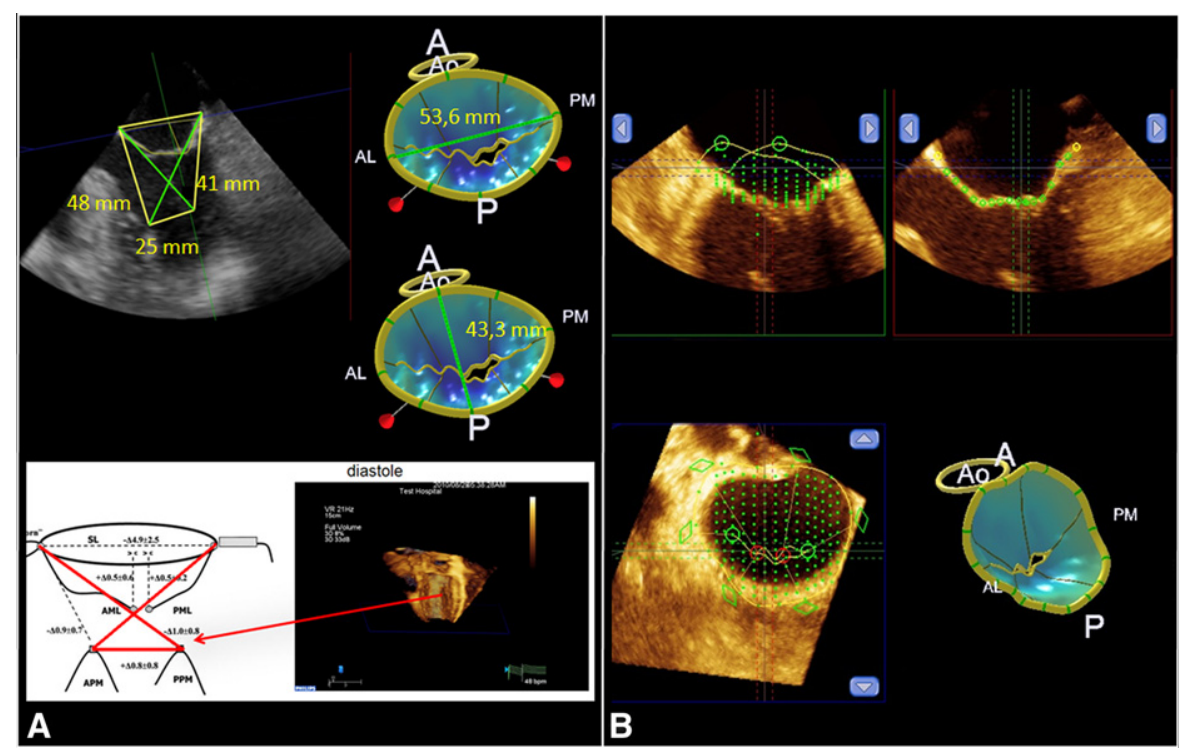

FIGURE 1. A, Mitral valve annulus diameters and distance between the tip of PPM and the ipsilateral annulus were measured on multislice systodiastolic views of the mitral valve apparatus and offline reconstruction by MQV software. B, The shape of the annulus and leaflets was traced. $A L$, Anterolateral; $P M$, posteromedial; $A o$, aorta; $S L$, septolateral; $A M L$, anterior mitral leaflet; $P M L$, posterior mitral leaflet; $A P M$, anterior papillary muscle; $P P M$, posterior papillary muscle. 


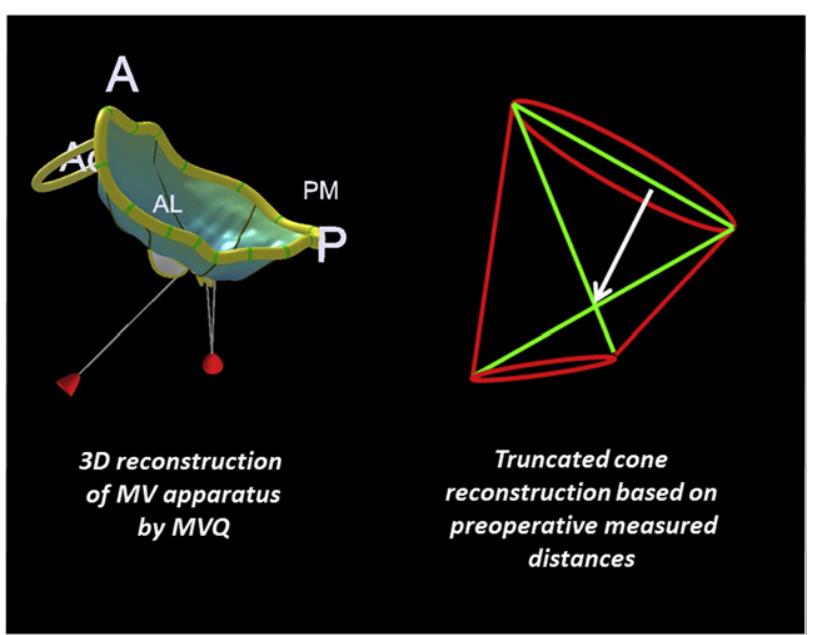

FIGURE 2. Schematic mitral valve apparatus model and the respective truncated cone were traced. $A L$, Anterolateral; $P M$, posteromedial; Ao, aorta; 3D, 3-dimensional.

tips of PPMs, and the distance between the tips of each PPM and the ipsilateral annulus.

Preoperative offline reconstruction of the expected MVA after annuloplasty. The prosthetic ring was selected according to the surface area of the anterior mitral leaflet measured preoperatively using the MVQ software (green zone). The free edge of the leaflet (red zone) was excluded because it represents the coaptation surface (Figure 3). A new expected truncated cone structure was traced considering the diameters of the selected prosthetic ring instead of the native valve annulus diameter (Figure 4). An expected new tenting area and coaptation depth (expected after annuloplasty) were obtained. Moreover, a normal coaptation depth (about $0.6 \mathrm{~cm}$ ) was considered as reference and traced into the new truncated cone. First we put into the new tenting area the length of the new desirable coaptation depth and then we drew 2 new diagonals (Figure 4). Thus, we calculated the new position of the PPM head that might be reached by relocation of the PPMs to reduce the tenting area and obtain a desirable coaptation depth. We measured the distance between the new position of the PPM tips and the previous position. This distance was equal to the amount of PPM relocation of each muscle. Finally, the distance between the plane of the annulus and the new position of each PPM head was calculated preoperatively and used during surgery as a reference to check the correct position of the PPMs after relocation. After surgery, all parameters were measured, recalculated, and compared with expected data (Figure 5).

\section{Surgical Technique}

The PPM relocation was performed using polytetrafluoroethylene (PTFE) CV-4 suture (Gore-Tex; W. L. Gore \& Associates, Inc, Flagstaff, Ariz) to reduce tenting area and coaptation depth. PPM relocation was performed by passing a PTFE suture through the fibrous portion of each PPM head reinforced by pledgets. Each needle of the double-armed suture was then passed up through the ipsilateral mitral annulus just between the fibrous trigone and the commissure. Two among the 3 heads (anterior and medial) of the posterior PPM were relocated by a couple of PTFE sutures. Mitral annuloplasty was then performed in all patients using a complete Edward Physio II annuloplasty ring (Edwards Lifesciences, Irvine, Calif) or a saddle rigid ring (St Jude Medical, Inc, St Paul, Minn). The size of the ring was selected preoperatively (based on its surface area given by the manufacturer) according to the surface area of the anterior mitral leaflet measured using MVQ. Interrupted 2-0 Ti-Cron U stitches (Ethicon, Inc, Somerville, NJ) were placed through the annulus and the prosthetic ring was seated. The PTFE sutures placed previously to the tips of both PPMs and subsequently through the mitral annulus were sutured to the ring and pulled up, drawing the PPMs tips closer to the annulus. The final position of both PPM tips (according to the distance measured and expected preoperatively using offline reconstruction) were estimated by measuring the length of the PTFE stitch from the PPM tips to the annulus. Finally, the sutures were tied. The adequacy of repair was tested using a saline solution to feel the LV.

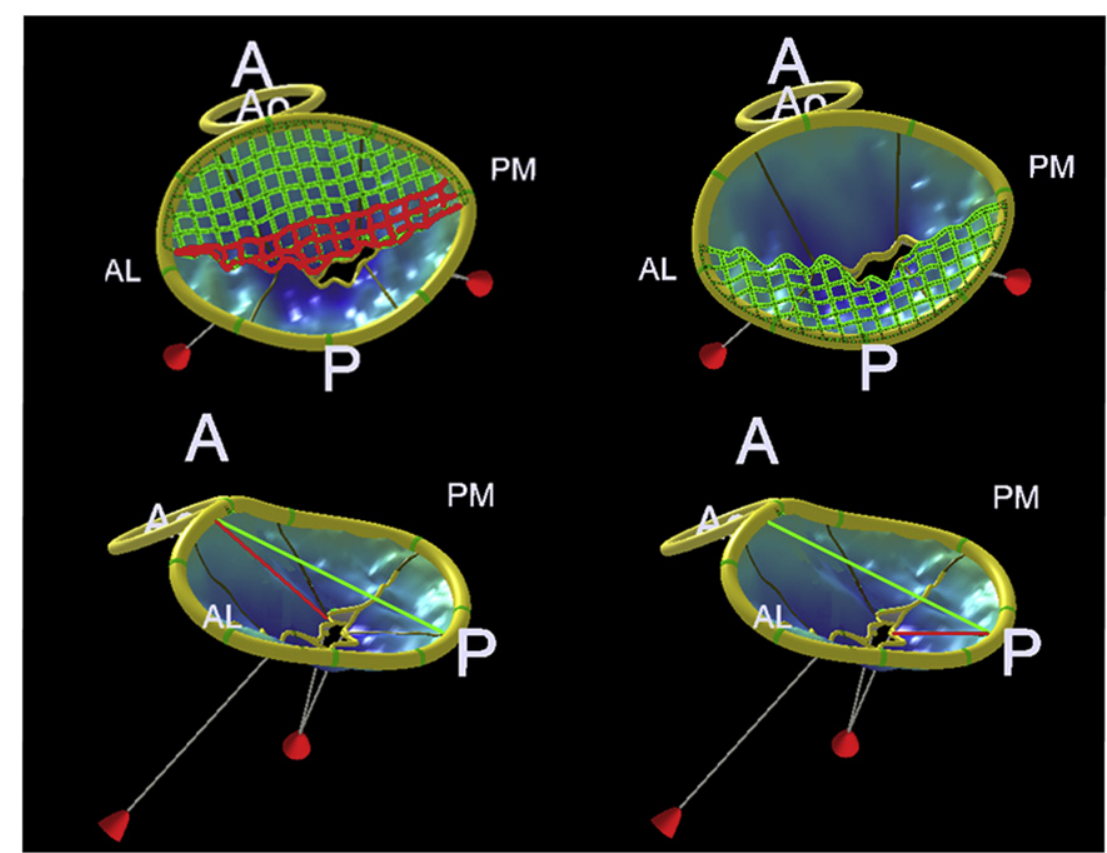

FIGURE 3. Surface of the leaflets and angle between the leaflets and the mitral annulus were calculated. $A L$, Anterolateral; $P M$, posteromedial; $A o$, aorta. 


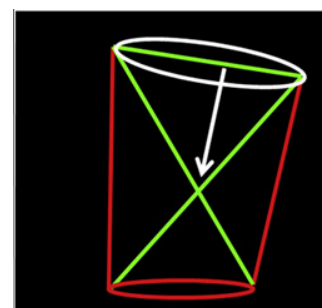

Reconstruction for expected truncated cone after mitral valve annuloplasty with

preoperative selected ring size.

Arrow indicates an expected new coaptation depth.

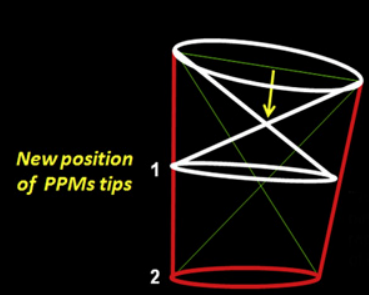

A desirable coaptation depth about $0,6 \mathrm{~cm}$ and 2 diagonals were traced. The distance between 1 and 2 represents amount of PPM relocation
FIGURE 4. Expected truncated cone was retraced according to the diameters of the selected ring despite the normal annular diameter; expected normal coaptation depth was used to obtain the expected position of the papillary muscle $(P P M)$ tips.

\section{RESULTS}

All patients had suitable real-time 3D TOE images for offline quantification of the MVA. The surface area of the anterior leaflet was obtainable in all patients. The mean time duration for image acquisition and offline reconstruction lasted $12 \pm 5$ minutes. No in-hospital death occurred. Four patients had trivial to mild residual MR postoperatively and a mean follow-up of $14 \pm 4$ months. The schematic reconstruction of the MVA using the QLab-MVQ software allowed the reconstruction of the truncated cone in all patients. After selection of the prosthetic ring size, the expected truncated cone geometry was obtained and the new position of PPM tips was detected. All expected diameters and distances of the MVA were measured and compared with postoperative data. No statistically significant difference was found between expected and postoperative intercommissural $(P=.35)$ and septolateral $(P=.89)$ annular diameters, coaptation depth $(P=.78)$, and the distances between the anterior $(P=.23)$ and posterior $(P=.34)$ PPM tip and ipsilateral annulus. All data are listed in Table 1.

\section{COMMENTS}

The optimal surgical treatment for FMR remains controversial. FMR is determined by complex mechanisms. LV remodeling with PPM displacement and leaflet tethering, reduced systolic leaflet closing force, dyssynchrony, and mitral annular enlargement are all contributors to FMR. ${ }^{7}$ Restrictive annuloplasty is the most commonly performed surgical procedure for FMR and provides good results in selected patients with minimal LV dilatation and mitral valve deformation. However, the persistence and recurrence of FMR in patients who had undergone restrictive annuloplasty remains high (up to $30 \%$ at 6 months postoperatively). Some authors have found that at 10-year follow-up persistent FMR can attenuate the potential survival benefit of a combined procedure compared with coronary artery bypass grafting alone (10-year survival for both being approximately $50 \%){ }^{8}$ The relatively high recurrence rate of FMR after restrictive annuloplasty has led to the development of new alternative surgical procedures targeting the subvalvular apparatus and the LV. The objective is to tailor the ideal combination of annular, valvular, chordal,

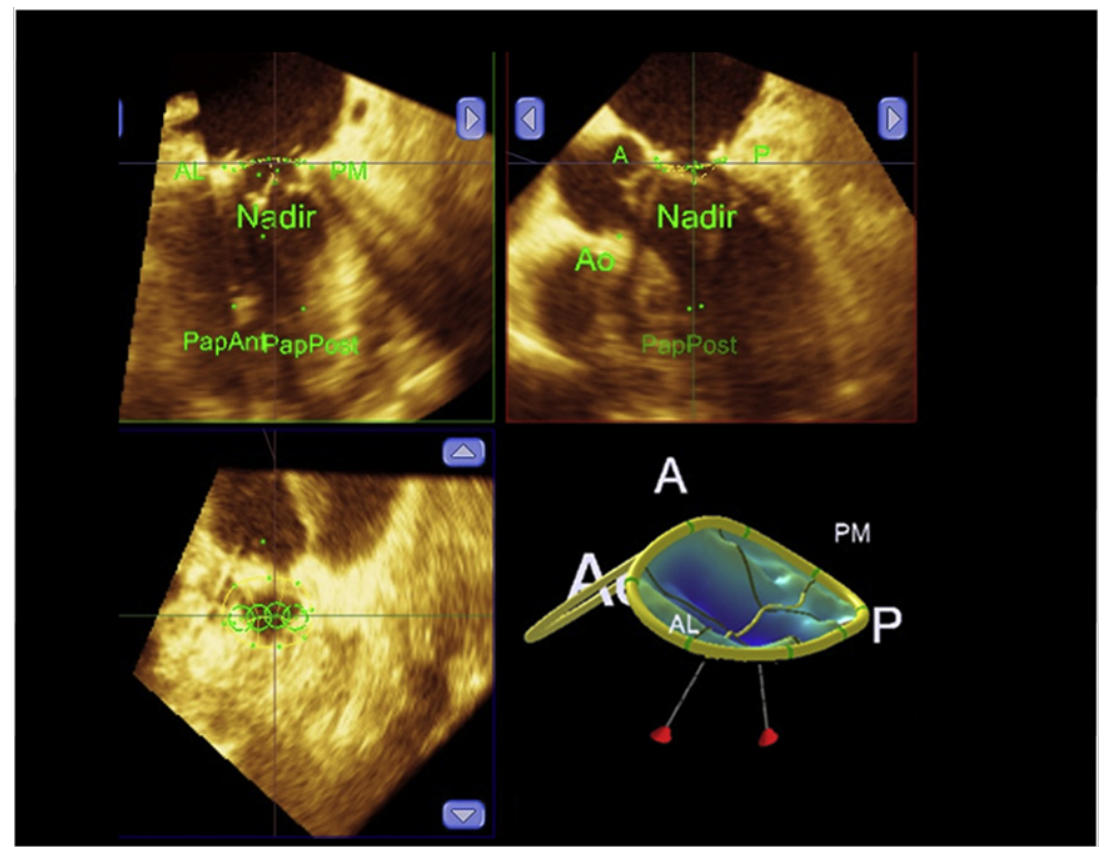

FIGURE 5. Postrepair parameter measurements. $A L$, Anterolateral; $P M$, posteromedial; $A o$, aorta; $A$, anterior; $P$, posterior. 
TABLE 1. Three-dimensional echocardiographic data: Before and after mitral valve surgery

\begin{tabular}{|c|c|c|c|c|}
\hline IMR 3D variables & Baseline data & $\begin{array}{c}\text { MVQ offline analysis expected } \\
\text { after annuloplasty }\end{array}$ & Postoperative data & $P$ value* \\
\hline \multicolumn{5}{|l|}{ Mitral annulus } \\
\hline Intercommissural distance & $51.8 \pm 1.2$ & $29 \pm 7$ & $30 \pm 3$ & .35 \\
\hline Septolateral distance & $44.8 \pm 0.7$ & $21 \pm 8$ & $21 \pm 2$ & .89 \\
\hline \multicolumn{5}{|l|}{ Mitral leaflets } \\
\hline Anterior leaflet area $\left(\mathrm{mm}^{2}\right)$ & $722.6 \pm 256.2$ & $411 \pm 61$ & $395 \pm 56$ & .52 \\
\hline \multicolumn{5}{|l|}{ Subvalvular distance (mm) } \\
\hline AL. PPM-ipsilateral annulus & $48 \pm 5$ & $34 \pm 5$ & $35 \pm 3$ & .23 \\
\hline PM. PPM-ipsilateral annulus & $41 \pm 3$ & $31 \pm 4$ & $30 \pm 2$ & .34 \\
\hline PPM tips* & $25 \pm 0.2$ & $20 \pm 3$ & $19 \pm 1.5$ & .42 \\
\hline \multicolumn{5}{|l|}{ Leaflet angles } \\
\hline Posterior leaflet angle $\left({ }^{\circ}\right)$ & $45.8 \pm 4.2$ & $37.2 \pm 5.2$ & $38.5 \pm 3.2$ & .12 \\
\hline Anterior leaflet angle $\left(^{\circ}\right)$ & $37.5 \pm 2.8$ & $33.2 \pm 3.1$ & $31.2 \pm 2.8$ & .08 \\
\hline \multicolumn{5}{|l|}{ Coaptation } \\
\hline Coaptation depth $(\mathrm{cm})$ & $1.3 \pm 0.2$ & $0.6 \pm 0.2$ & $0.55 \pm 0.2$ & .78 \\
\hline \multicolumn{5}{|l|}{ Tethering } \\
\hline Tenting area $\left(\mathrm{cm}^{2}\right)$ & $3.2 \pm 0.5$ & - & $1.2 \pm 0.2$ & - \\
\hline Tenting volume $(\mathrm{mL})$ & $8.9 \pm 1.7$ & - & $1.4 \pm 0.3$ & - \\
\hline
\end{tabular}

$I M R$, Ischemic mitral regurgitation; $A L$, anterolateral; $P M$, posteromedian; $P P M$, papillary muscle. * Significance between expected and postoperative data.

PPM, and ventricular relationships on the basis of preoperative clinical and echocardiographic characteristics to achieve the best repair result in each patient. ${ }^{9}$ This will lead to a "continuum of therapies" that can be customized to the individual patient's needs. In the present study, we have reported, for the first time, an initial step toward a patient-specific therapeutic approach to FMR using preoperative 3D reconstruction of the MVA complex with the MVQ software. The complex 3D relationship between the PPMs and the mitral annulus was revealed as a truncated cone. After adjustment to the measured preoperative ring size and referring to a normal coaptation depth, the new positions of the PPM heads were obtained and taken into account for the surgical intervention. After PPM repositioning, this complex 3D PPM-annulus geometric shape was retraced; it mimics the preoperative retraced one as a whole. Our data highlighted that PPM relocation and mitral valve annuloplasty could be oriented by preoperative $3 \mathrm{D}$ echocardiography and offline analysis using the MVQ software. Furthermore, with increased clinical experience, this approach might adequately predict the surgical gestures.

We thank Dr Roberto Francavilla for the technical assistance in image acquisition.

\section{References}

1. Bolling SF, Deeb GM, Brunsting LA, Bach DS. Early outcome of mitral valve reconstruction in patients with end-stage cardiomyopathy. $J$ Thorac Cardiovasc Surg. 1995;109:676-82.

2. McGee EC, Gillinov AM, Blackstone EH, Rajeswaran J, Cohen G, Najam F, et al. Recurrent mitral regurgitation after annuloplasty for functional ischemic mitral regurgitation. J Thorac Cardiovasc Surg. 2004;128:916-24.

3. Crabtree TD, Bailey MS, Moon MR, Munfakh N, Pasque MK, Lawton JS, et al. Recurrent mitral regurgitation and risk factors for early and late mortality after mitral valve repair for functional ischemic mitral regurgitation. Ann Thorac Surg. 2008;85:1537-43.

4. Kron IL, Green GR, Cope JT. Surgical relocation of the posterior papillary muscle in chronic ischemic mitral regurgitation. Ann Thorac Surg. 2002;74:600-1.

5. Vergnat M, Jassar AS, Jackson BM, Ryan LP, Eperjesi TJ, Pouch AM, et al. Ischemic mitral regurgitation: a quantitative three-dimensional echocardiographic analysis. Ann Thorac Surg. 2011;91:157-64.

6. Jensen H, Jensen MO, Smerup MH, Ringgaard S, Sørensen TS, Andersen NT, et al. Three-dimensional assessment of papillary muscle displacement in a porcine model of ischemic mitral regurgitation. J Thorac Cardiovasc Surg. 2010;140: 1312-8.

7. Sonne C, Sugeng L, Watanabe N, Weinert L, Saito K, Tsukiji M, et al. Age and body surface area dependency of mitral valve and papillary apparatus parameters: assessment by real-time three-dimensional echocardiography. Eur J Echocardiogr. 2009;10:287-94

8. Ryan LP, Jackson BM, Hamamoto H, Eperjesi TJ, Plappert TJ, St John-Sutton M, et al. The influence of annuloplasty ring geometry on mitral leaflet curvature. Ann Thorac Surg. 2008;86:749-60.

9. Bouma W, van der Horst IC, Wijdh-den Hamer IJ, Erasmus ME, Zijlstra F, Mariani MA, et al. Chronic ischaemic mitral regurgitation. Current treatment results and new mechanism-based surgical approaches. Eur J Cardiothorac Surg. 2010;37:170-85. 